\title{
Water vapor transport in soils from a pervaporative irrigation system
}

\author{
Lindsay C. Todman ${ }^{1}$, Andrew M. Ireson ${ }^{2}$, Adrian P. Butler ${ }^{3}$ and Michael R. Templeton ${ }^{4}$
}

\section{ABSTRACT}

A novel method for irrigation with saline water uses a polymer membrane, formed into a tube, to treat and distribute the water simultaneously. The flux of water across the membrane occurs by the process of pervaporation, during which a phase change from liquid to vapor occurs. Thus water arrives in the soil in vapor phase. The experimental results presented in this paper demonstrate that, contrary to previous assumptions, soil vapor flows are a significant transport mechanism during pervaporative irrigation in dry soils. The soil water sorption properties affect the rate of condensation in the soil, which in turn affects both the water distribution in the soil and the loss of water vapor to the atmosphere. The flux from the tube becomes limited by high humidities adjacent to the external surface of the membrane. Thus enhancing condensation in the soil or increasing diffusion through the soil increases flux from the system. These findings highlight the need to consider how plants might interact with water supplied in the vapor phase.

\footnotetext{
${ }^{1} \mathrm{PhD}$ Student, Dept. of Civil and Environmental Engrg., Imperial College London, South Kensington, London, SW7 2AZ, UK. E-mail: lindsay.todman08@imperial.ac.uk

${ }^{2}$ Assistant Professor, University of Saskatchewan, Saskatoon, Saskatchewan, S7N 3H5, Canada

${ }^{3}$ Reader, Dept. of Civil and Environmental Engrg., Imperial College London, South Kensington, London, SW7 2AZ, UK.

${ }^{4}$ Senior Lecturer, Dept. of Civil and Environmental Engrg., Imperial College London, South Kensington, London, SW7 2AZ, UK.
} 
Keywords: Irrigation; soil vapor flow; membranes; pervaporation; salinity

\section{Introduction}

With growing pressure on the availability of freshwater for agriculture, irrigators are increasingly exploiting lower quality water sources (Pereira et al., 2002). However irrigating with these waters can, over time, contribute to soil salinization (Penov et al., 2011) or cause other environmental concerns (Beltrán, 1999). The pervaporative irrigation system used in this research is described previously in a series of publications (Quiñones-Bolaños et al., 2005a,b; Quiñones-Bolaños and Zhou, 2006). This system provides in-situ treatment of saline water while simultaneously supplying it to the plant root zone. To irrigate in this way a pervaporative polymer membrane is formed into a tube, buried in the ground and filled with saline water. When the surrounding soil is dry a chemical potential gradient exists across the membrane and draws water into the soil, whilst the transport of salt is limited. The water flux occurs via a membrane transport process called pervaporation. As plants take up water from the root zone the soil moisture content is reduced, decreasing the chemical potential in the soil. This maintains a gradient across the tube, which enables the continued transport of water. The system is intended for use in arid regions, generally deserts, where freshwater is limited but there is an available supply of saline water.

Membrane transport processes like reverse osmosis, gas permeation and pervaporation are frequently used in separation technologies (Pabby et al., 2008). Pervaporation is distinct these other membrane transport processes because of the phase change from liquid to vapor that occurs during the process (Feng and Huang, 1997). Conceptually the process of pervaporation is often considered in three steps:

\section{Sorption of the permeate into the membrane}


2. Diffusion of the permeate across the membrane

3. Desorption of the permeate in the vapor phase at the external edge

Transport across the membrane used in this research occurs by pervaporation because the membrane polymer is highly hydrophilic. Thus molecules of water are readily adsorbed into the polymer but desorb from it primarily in the vapour phase. Mathematically, the process of pervaporation can be modeled using the solution-diffusion equation (Paul, 2004), which takes into account the sorption of the permeate into the membrane and its subsequent diffusion across the membrane. The exact location of the phase change from liquid to vapor is unknown, thus diffusion across the membrane may occur in either liquid phase, vapor phase or both. To simplify calculations it is often assumed that transport occurs entirely in one phase. Thus the driving chemical potential gradient is calculated either using a liquid concentration gradient or, more commonly, a vapor pressure gradient applied across the membrane (Wijmans and Baker, 1995). Sumesh and Bhattacharya (2006) suggest that transport through the membrane occurs entirely in liquid phase if the gradient across the membrane is below a threshold value. However as the applied gradient increases the liquid-vapor interface retreats into the membrane, away from the external surface.

The efficacy of a pervaporative irrigation system to treat saline water has already been a subject of some study (Quiñones-Bolaños et al., 2005a,b). From a water treatment perspective a particular benefit of the system is its low energy requirement, as the driving force for the water flux is provided by the environmental conditions surrounding the tube. As an irrigation system, another feature of the system also stands out; the inherent feedback between the crop water uptake and the irrigation flux. In recent years techniques such as irrigation scheduling (Jones, 2004) and precision irrigation (Sadler et al., 2005) have been developed to 
contrive such feedback mechanisms. These methods use sensors to monitor plant water stress (either directly or indirectly). A control system is then implemented to apply water as required, both spatially and temporally. In pervaporative irrigation the system automatically responds to the soil moisture conditions without the need for monitoring. However, once the pervaporative tube is in operation the user has no control over the flux rate. Thus it is important to ensure that sufficient membrane surface area is present in the soil by estimating the likely flux rate from the tube.

One model (Quiñones-Bolaños and Zhou, 2006) currently exists to predict the flow rate across the irrigation tube in the soil. In this model the soil moisture conditions are simulated mathematically so that the feedback between the soil moisture conditions and the flux from the tube can be represented. However, it is assumed that the mass transport of water through the soil only occurs in the liquid phase. As the water leaves the membrane in vapor phase it is thus assumed that all of the mass permeating through the tube condenses in the near vicinity. However, it is possible that vapor transport through the soil affects both the distribution of the liquid soil water content and the mass transfer of water to the atmosphere at the soil surface.

Diffusive vapor transport through soil occurs due to gradients in the partial pressure of water vapor in the soil pores. Such gradients can occur due to variations in temperature, solute concentration and soil water content, all of which affect the equilibrium relativity humidity between the liquid and vapor phases in the soil. Gradients in temperature (Phillip and de Vries, 1957; Bittelli et al., 2008) and solute concentration (Kelly and Selker, 2001) are often considered to be significant near to the soil surface under field conditions. However, in this research, no significant gradients in temperature or solute concentration are expected due to the experimental methods used. Instead, because of the dry soil 
conditions, partial pressure gradients that occur due to variations in soil water content are of particular interest.

In general, vapor flows due to the variation in soil water content are small because the vapor pressure in most soils approaches the saturated vapor pressure at soil water contents above the residual water content of the soil. This can be surmised by observing that, in the absence of osmotic effects, the equilibrium humidity in soil is theoretically greater than $99.5 \%$ even at a suction pressure of 6 bar (Hillel, 1998, p150). Such high suction pressures correspond to low water contents thus vapor pressure gradients due to soil water content variations are often small. It has been suggested that the residual water content (although often used as a fitting parameter) represents the water content below which water is retained in the soil primarily by adsorptive forces (Lebeau and Konrad, 2010). Hence, at water contents below this residual value, the water is no longer held in the soil by capillarity but by short range adsorptive forces that bind water molecules to the surface of solid particles, forming liquid films (Churaev, 2000, p29-31). In these dry conditions bulk connectivity of water in the liquid phase breaks down and water transport can occur by two mechanisms; liquid film flow along the solid surfaces and vapor flow through the connected air phase (Churaev, 2000, p123). Significantly, when water is retained in the soil by adsorptive forces, the equilibrium relative humidity of the vapor phase decreases (Ruiz and Benet, 2001) thus vapor pressure gradients can become significant even for tiny variations in soil water content and isothermal vapor flow can occur. Although water contents below the residual value are not common in field soils they do occur in arid and semi-arid environments as the atmospheric conditions are very dry and the soil moisture content near the soil surface is often in the adsorptive range (Agam and Berliner, 2006), approaching equilibrium with the dry surrounding air. 
As this irrigation system is intended for use in arid, desert regions it is therefore likely that vapor flows due to variations in soil water content will occur. The experimental method used in this study was designed to re-examine the assumption made in previous work (Quiñones-Bolaños and Zhou, 2006) that water transport through soil can be considered entirely in the liquid and to consider its validity in different environmental conditions. The focus is on developing an understanding of the physical processes, specifically the vapor flow, that affect the irrigation flux into the soil and quantifying the flux in various environmental conditions. To simplify the analysis this work was carried out in bare soil, without the presence of a crop.

\section{Experimental methods}

The two experimental setups used in this research are shown in Figure ??. The air box setup was designed to quantify the flux from the pervaporative membrane under different humidity conditions, i.e. under differing vapor pressure gradients, without the complications of transport through the soil. The soil box experiments were designed to explore how the presence of the soil, and various soil conditions (soil depth, soil type, soil salinity, atmospheric humidity), affect the flux, and how moisture is transmitted through and retained in the soil in liquid and vapor phases. Furthermore, to aid with the interpretation of the soil box experiments, moisture sorption isotherms were determined to quantify the water content of the soils at equilibrium in different humidity conditions. All of the experimental work was carried out in a laboratory maintained at $21 \pm 1^{\circ} \mathrm{C}$. The pervaporative membrane used in these experiments was a non-porous hydrophilic polymer composed of a thermoplastic block copolymer of the polyester family synthesised by Du Pont de Nemours (Geneva, Switzerland). The dry thickness of the membrane was $0.75 \mathrm{~mm}$. The polymer was extruded into tubular form and 

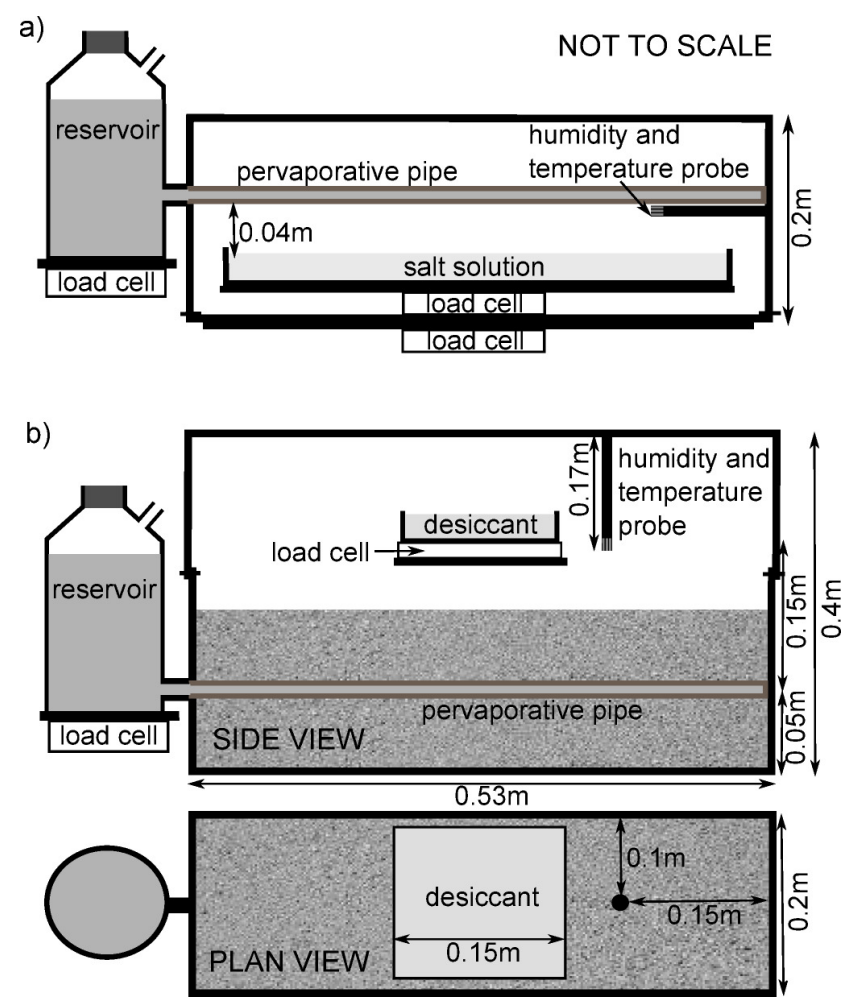

FIG. 1. Diagrams of the experimental setups for a) air box tests in which the tube was enclosed in a humidity chamber with conditions created using a saturated salt solution b) soil box tests in which the tube was buried in soil.

corrugated (Figure ??) to provide structural strength. The inner diameter of the tube was $19 \mathrm{~mm}$ and the outer diameter was $23 \mathrm{~mm}$ due to the corrugations.

Air box tests

The air box setup (Figure ??a) was constructed to enclose the tube within an air filled chamber so that the flux under different humidity conditions could be observed. The initial conditions in the chamber were established using a saturated salt solution, which maintained the relative humidity at a constant level. Once the pervaporation tube was filled with water the humidity increased as moisture evaporated from the tube, diffused through the air and condensed into the salt solution. A series of saturated salt solutions were used to maintain the 
humidity at different levels. These saturated salt solutions were; lithium chloride (11\%), calcium chloride (33\%), magnesium chloride (37\%), calcium nitrate $(55 \%)$, sodium chloride (75\%) and potassium chloride (85\%). The relative humidities given in brackets indicate the equilibrium condition between a saturated salt solution and air at $21^{\circ} \mathrm{C}$.

The pervaporative tube was stretched across the length of the humidity chamber and clamped at the entry and exit points by cable glands. Eighty corrugations of tube were within the box corresponding to a dry, un-stretched length of $34 \mathrm{~cm}$. The corrugations passing through the length of the cable gland were wrapped in polyfilm to prevent pervaporation from this surface area. Outside of the chamber the tube entered a PVC tube sealed with silicone sealant. One end of this tube was connected to a supply reservoir placed on a load cell, whilst the other end was bunged. Thus the water in the tube was in approximately hydrostatic conditions, other than the small flow rate due to the pervaporative flux from the tube. The polypropylene box that formed the humidity chamber was sealed around the lid with a foam sealant strip, clamped closed and placed on a load cell. Cables exiting the box were sealed using cable glands. A tray containing a saturated salt solution, and with excess salt, was positioned $4 \mathrm{~cm}$ below the tube and placed on a load cell to monitor the mass. The load cells were supplied by Applied Measurements Ltd (Aldermaston, UK). The cells for the reservoir and the salt solution had a $3 \mathrm{~kg}$ maximum load (specification OBUG-3kg), whilst the mass of the chamber was monitored using a cell with a $10 \mathrm{~kg}$ maximum (OBUG-10kg). All of the cells were fitted with aluminum platforms of appropriate dimensions. The temperature and humidity probe was supplied by Michell Instruments (Ely, UK), specification PC33-3-XX-T3-C (accuracy \pm 3 for $30-80 \% \mathrm{RH}$ ).

The chamber was left for 24 hours before the start of the experiment to allow it to reach a pseudo-equilibrium condition. A temperature and humidity probe 
positioned below the tube monitored the state of the chamber. To start the test a tap between the supply reservoir and irrigation tube was opened, allowing the tube to fill with water. The bung at the far end of the tube was removed to purge air from the system and replaced when the tube was filled with water. Thus some of the water exiting the supply reservoir at this time did not remain in the tube. At the start of the experiment data were collected for one hour at one minute intervals and for a further three hours at five minute intervals. For ten hours before the irrigation started, and for the rest of the duration of the experiments, the data were collected at fifteen minute intervals. The data were collected using a National Instruments (Newbury, UK) NI USB-6210 data logger connected to the LabVIEW software from the same supplier. A single ended voltage measurement was made for each sensor as they all had a common ground. Each data point was collected by sampling at a frequency of $10 \mathrm{kHz}$ for two seconds and recording the mean and standard deviation of the measurement.

\section{Soil box tests}

Figure ??b shows the soil box setup used for experiments to quantify the flux of water from the tube into dry soil. The setup was similar to the air tests described above but the humidity chamber was twice the size and the irrigation tube was buried in soil. The same load cells were used to monitor the reservoir and desiccant mass as for the air tests. The combined humidity and temperature probe was in the air gap between the soil and the desiccant, positioned as in the diagram. This soil box setup was used to conduct three sets of tests; one set with varying depths of sand, one set with different conditions in the humidity chamber and a final set with three different soil types. In the first set the depth of the sand in the box was varied. The minimum depth was $7 \mathrm{~cm}$ (from the base of the box) and the maximum was $15 \mathrm{~cm}$. The tube was consistently buried $5 \mathrm{~cm}$ from the 


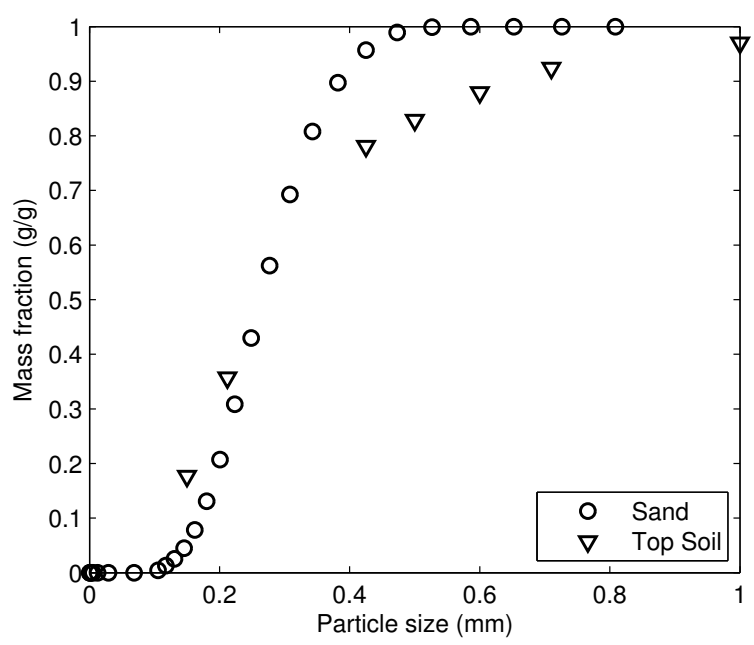

FIG. 2. Particle size distribution of the sand and top soil. The particle size of the sand was determined by lazer diffraction using a Coulter LS100 (Beckman Coulter, Inc., High Wycombe, UK), as the density of some particles was close to that of water the particle size was determined by sieving.

base of the box. All of these experiments were conducted using sand and $100 \mathrm{~g}$ of a calcium chloride based desiccant (brand name 'Drysac', Superdry Superior Container Desiccant, Singapore). For comparison, an additional experiment was performed using the same desiccant but without any soil. In the second set of experiments the conditions in the humidity chamber were varied. One experiment was conducted without a desiccant in the box, one with the 'Drysac' desiccant, another with a saturated calcium chloride salt solution (instead of the desiccant) and a final test in which the lid was removed from the box and the surface was exposed to the ambient laboratory conditions. This set of experiments was performed in sand with a depth of $15 \mathrm{~cm}$. In the third set of experiments three types of soil were used; marine sand, a garden top soil and a salinized sand. Data on the properties of these soils are provided in Figure ??, Figure ?? and Table ??. The saline sand consisted of marine sand with an added $16 \mathrm{~g}$ of sodium chloride per kilogram of sand. In this set of experiments there was no desiccant present in the box and the soil was packed to a depth of $10 \mathrm{~cm}$. 

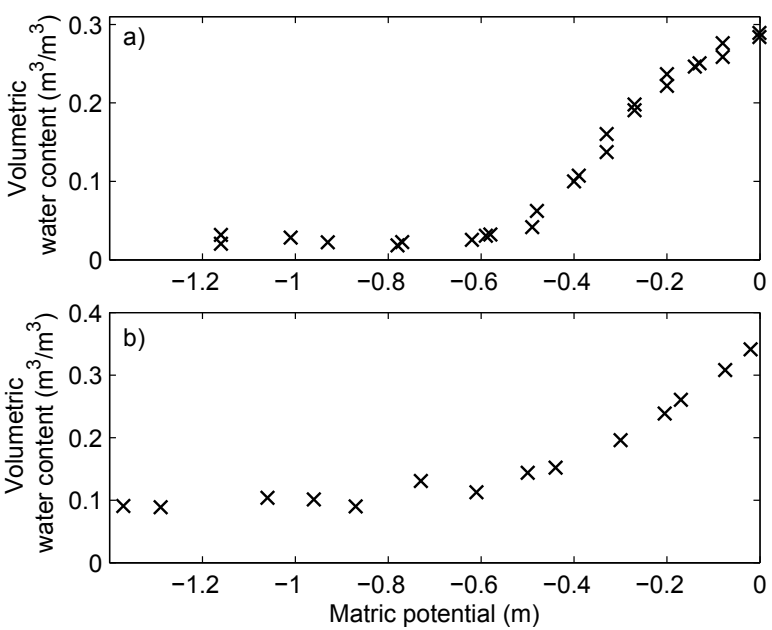

FIG. 3. Soil water retention characteristic of a) sand and b) top soil. These data were collected by placing a sample of the soil (packed at the target density) on a porous plate and applying a suction pressure to the underside of the plate using a hanging column of water (Haines, 1930). The water content of each soil sample was then determined gravimetrically. From this data the residual water content can be estimated as approximately $0.02 m^{3} / m^{3}$ in sand and $0.08 m^{3} / m^{3}$ in top soil.

Before each experiment the soil was dried in an oven at $105^{\circ} \mathrm{C}$ for 24 hours and stored in an air tight container with silica gel desiccant to cool. Although this procedure reduced the soil water content far beyond the permanent wilting point (generally considered the minimum water content at which plants can grow), this dryness represented possible field conditions in an arid or semi-arid environment. The soil was packed into the boxes $1 \mathrm{~kg}$ at a time and was compacted with a flat aluminum pestle. The soil surface was then lightly scarified to improve the hydraulic connection with the next layer, following the method reported by Lewis and Sjöstrom (2010) for dry soil packing. After some of the experiments, samples were taken from the soil to determine the liquid soil moisture content. Two samples, each weighing approximately 200g, were taken from each box. These samples were taken from the top $1 \mathrm{~cm}$ of the soil and from the region immediately surrounding the PV membrane (i.e. less than $1 \mathrm{~cm}$ from the membrane). The soil 
water content was determined gravimetrically by weighing the sample, drying it in an oven at $105^{\circ} \mathrm{C}$ for 24 hours, cooling it in a desiccator and re-weighing the sample. Gravimetric water contents were converted to the equivalent volumetric water content using the packing density of each of the soil types (Table ??).

The moisture sorption isotherms for the different soil types were determined by two methods; desiccator experiments and using a vapour sorption analyzer (VSA). Desiccator experiments were performed by enclosing soil samples in a series of desiccators (Dexter and Richard, 2009). Samples were approximately $100 \mathrm{~g}$ in weight. The relative humidity was maintained in each desiccator by a saturated salt solution. The samples were allowed to reach equilibrium, a process which took between 2-6 weeks. The water content of each sample was then determined gravimetrically. VSA experiments were performed by Labcell Ltd (Alton, UK) using a Decagon Devices (Pullman, WA, USA) analyzer. This device has a chamber in which a soil sample is placed. The humidity in the chamber is changed incrementally and the mass of the sample is monitored. The measurement limits are between 10-90\% relative humidity and the device monitors the change in mass rather than the absolute mass. Thus the results from the desiccator experiments were used to express the VSA results on an absolute scale.

\section{Results and discussion}

Air box tests

Figure ?? shows a sample of raw data that were collected in the course of these experiments. The initial change in the masses of the reservoir and soil box (observed at $\mathrm{t}=0 \mathrm{hrs}$ ) corresponded to the filling of the irrigation tube. Transient conditions were evident for approximately the next eight hours. During this time the relative humidity in the chamber increased, the temperature near to the tube decreased and the measured masses of the reservoir and soil box changed at a 

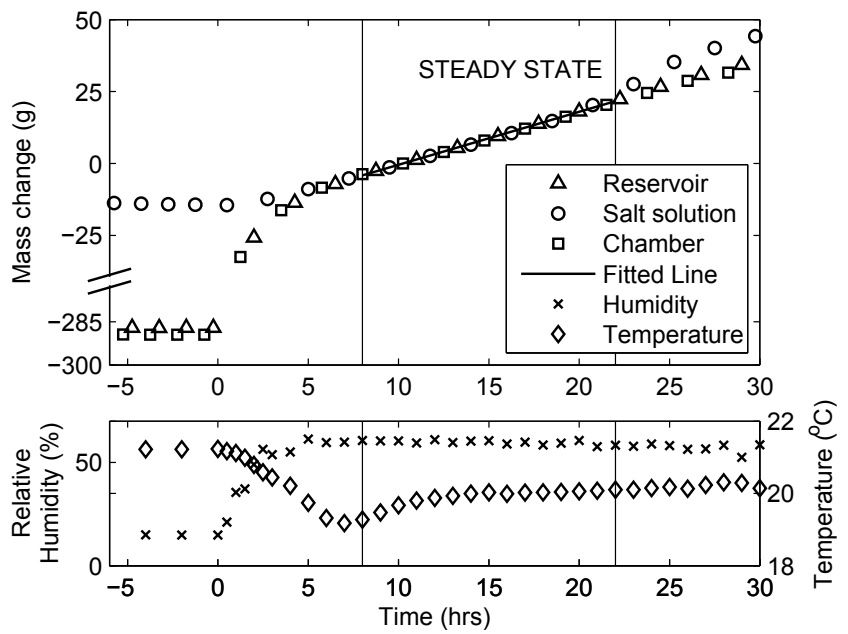

FIG. 4. Results for the air test experiments using a Lithium Chloride salt solution. The change in mass measured by the three load cells is shown relative to the measured value at $t=10 \mathrm{hrs}$. The mass change of the reservoir is plotted as a positive change to allow comparison with the soil box data.

faster rate than that of the salt solution. After this time the system reached a quasi-steady state in which the mass of water pervaporating from the tube was equal to that adsorbed by the salt solution. In this state a humidity gradient between the tube surface and the surface of the salt solution maintained diffusive transport through the air. The system was not strictly at steady state as the excess salt in the salt solution was constantly dissolving, but this effect was small during the test period as excess salt was provided. Thus, a quasi-steady state was maintained in the chamber. Slight variations in temperature (following ambient laboratory conditions) continued to have a small effect on the relative humidity in the chamber.

During the transient phase of the experiment the mass of water stored in the air increased and this was seen as an increase in relative humidity in the chamber. However, the mass of water in the air was only of the order of a few grams and did not explain the difference between the change in mass of the reservoir and the desiccant. The additional storage can be explained by membrane swelling, 
which occurred due to the sorption of water into the tube. This swelling increased the diameter of the tube and thus also increased the storage capacity for liquid water inside the tube. This additional capacity was filled from the reservoir and explains the difference in the mass change rate of the reservoir and the desiccant in the first five hours of the test. The length of the tube also increased due to the swelling; to minimize the effect of this the tube was stretched in its dry state before being clamped in position.

The flux rate from the tube in each experiment was estimated by fitting a linear relationship to the time series of reservoir mass when the system was in a quasi-steady state. This is illustrated in Figure ??. The data from the reservoir mass was selected as this was less sensitive to noise. Linear regression was performed using the MATLAB function 'robustfit', implemented using iteratively reweighted least squares with a bisquare weighting function. This function also provided an estimate of the parameter error. The calculated gradient gave the estimate of the mass flow rate, the error in predicted value of these gradients was small and negligible compared to errors in relative humidity. To convert to a flux rate it was assumed that the tube was equivalent to a cylinder $53 \mathrm{~cm}$ in length with a diameter of $2.6 \mathrm{~cm}$ (corresponding to the external diameter of the swollen tube). The mean and standard deviation of the relative humidity measured in the chamber was also calculated. For each of the salt solutions tested two experiments were conducted. The flux rates for each experiment were calculated individually and are shown in Figure ??

Figure ?? shows that the flux across the pervaporative membrane varied significantly with the surrounding partial vapour pressure as indicated by the relative humidity at $21^{\circ} \mathrm{C}$. For relative humidities greater than $75 \%$ (at the location of the probe) the relationship between the humidity and the flux appeared to vary linearly, however the result for the lithium chloride solution (in which the relative 


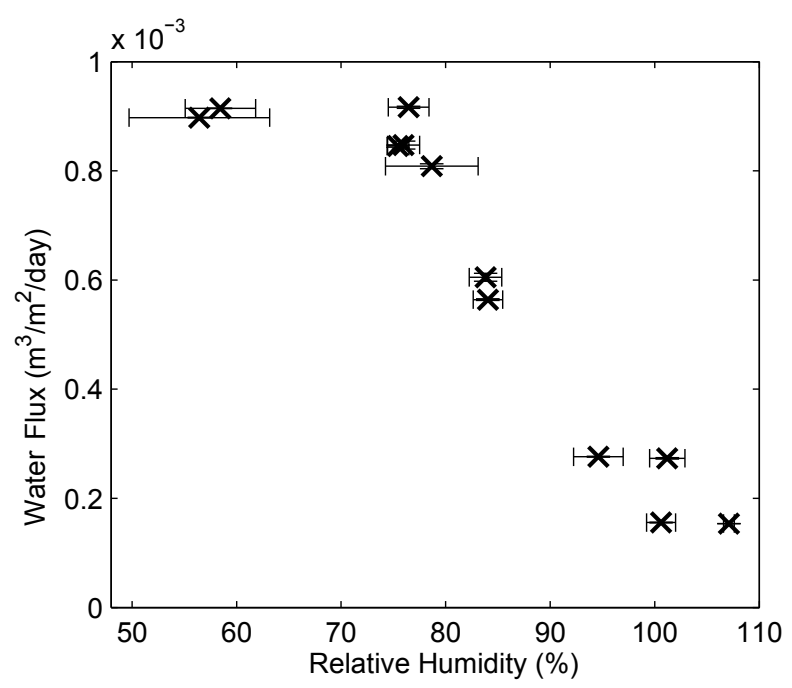

FIG. 5. Relationship between the observed flux and humidity in air tests.

humidity reached approximately 60\%) did not continue this trend. It is considered that the humidity measurements greater than $100 \%$ were likely to have occurred due to probe error in highly humid conditions. Note that despite the unexpected high humidity, the fluxes were consistent with those in the duplicate experiments. A small flux was observed even at a relative humidity of approximately $100 \%$. In these conditions, under the action of diffusion alone, the partial vapour pressure close to the membrane should be saturated and no flux would be expected across the membrane. It is possible that this flux was observed due to temperature effects, as the temperature at the membrane surface was slightly reduced by the evaporation of water. The slightly cooler, denser air should therefore slowly sink, transporting water vapor with it.

When the relative humidity is close to $100 \%$ it is likely that the phase change from liquid to vapor occurred at the external surface of the membrane. The flux rate was then limited by the rate of diffusion of water vapor away from the membrane surface. In experiments using salt solutions with lower equilibrium humidities the maximum possible partial pressure gradient was increased, thus 


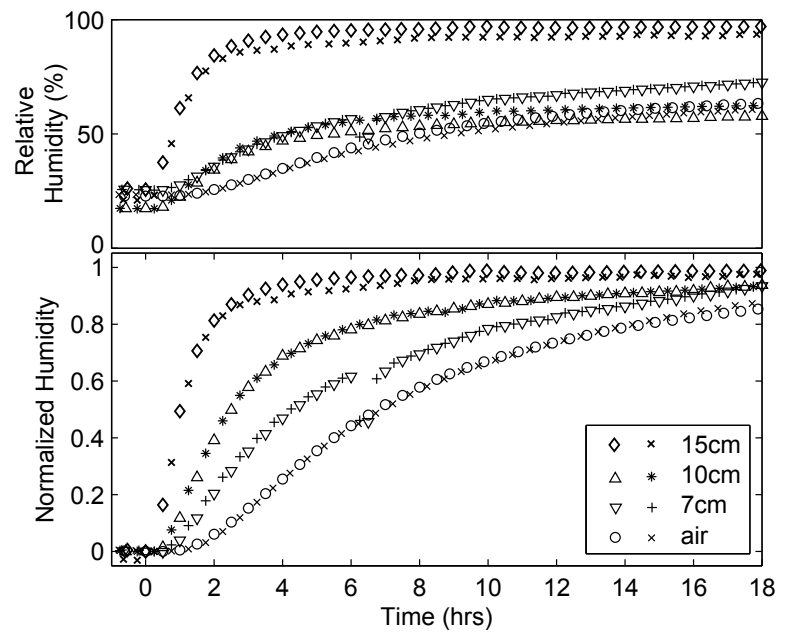

FIG. 6. Humidity change in the chamber after irrigation was commenced in sand of three different depths (from the base of the chamber) and in air. Duplicates of each experiment are shown. Note that in both of the tests with $10 \mathrm{~cm}$ a sudden increase in laboratory temperature $6.5 \mathrm{hrs}$ into the experiments caused a temporary decrease in humidity.

the rate of diffusion increased. However, as the humidity decreased further to below $60 \%$, the location of the phase change may have retreated away from the external edge of the membrane as suggested by Sumesh and Bhattacharya (2006). It is possible that such an effect could have a highly non-linear influence on the flux rate. The observation that the tube was visibly less swollen in the test using lithium chloride compared to the other tests provides qualitative support for this suggestion.

Overall, the results from the air box experiments demonstrated the magnitude of the vapor flux that can be achieved in humid conditions and show, as expected, that there is an inverse relationship between the partial vapor pressure (as indicated by the relative humidity) and the flux rate.

\section{Soil box tests}

Figure ?? shows the rate of change of the relative humidity in the first set of soil box tests, in which the depth of the sand was varied. For comparison, an 
additional experiment was performed in air alone. The humidity was 'normalized' compared to the initial and final values in the chamber thus the plotted 'normalized' humidity $\left(R H_{n}\right)$ can be expressed as

$$
R H_{n}=\left(R H-R H_{0}\right) /\left(R H_{40}-R H_{0}\right)
$$

where $R H_{0}$ is the initial relative humidity and $R H_{40}$ is the relative humidity 40 hours after the start of the test. This was done primarily because the commercial desiccant that was used in the test did not reliably maintain the humidity in the chamber at the same value between repeated tests, but the rate at which the humidity changed between the initial to the steady state conditions was repeatable.

When the experimental system was implemented in air it was clear that all mass transport occurred in the vapor phase. The presence of the sand decreased both the magnitude and speed of the humidity response in the chamber. As the depth of the sand was increased the rate of change of the humidity in the chamber decreased. The form of these breakthrough curves suggests that a diffusive process still dominated mass transport, although with a lower diffusion coefficient.

A time series for the flux rate of water into the soil was estimated by approximating the rate of change of the mass of the reservoir. To reduce the effect of noise (some of which was diurnal) the rate of change was approximated using the data from a 24 hour period. The flux rates were approximated as before using the MATLAB function 'robustfit'. Due to the chosen measurement window the first approximation could be only made at $\mathrm{t}=12 \mathrm{hrs}$. However, as the reservoir mass is affected by the tube swelling, the flux approximated in the initial stages of the test is artificially high, and should be neglected for at least the first 20 hours.

Figure ?? shows the results for the second set of soil box experiments in which the conditions at the soil surface were varied. The median, interquartile range and 


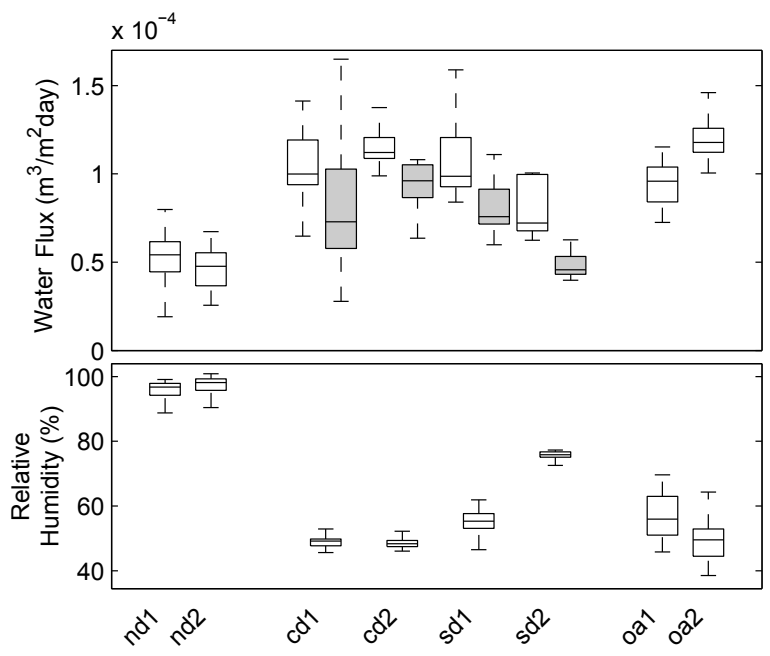

FIG. 7. The effect of environmental conditions on the irrigation flux. Four experiments are compared; one with no desiccant (nd), another with a calcium chloride salt solution (cd), the third with the commercial desiccant 'Drysac' (sd) and the fourth in a chamber without a lid, left open to the atmosphere (oa). The results for an independent repeat of each experiment are also shown. In the flux plot the flux computed from the change in the reservoir mass is shown in black and that computed from the change in the desiccant mass is shaded in gray.

range of the fluxes and relative humidity are shown, outliers are neglected. All of these experiments are performed in sand of $15 \mathrm{~cm}$ depth. Without a desiccant the flux across the PV membrane was lower than the flux when a desiccant was present. However, when a desiccant was present, a significant proportion of the water traveled through the sand and was adsorbed into the desiccant. Thus this water was not stored in the soil. This demonstrates that without considering the vapor flow the soil moisture available for plant uptake cannot be predicted. This finding is confirmed in the last test when the lid was removed from the soil box. The flux out of the tube was comparable to that in the tests with a desiccant in the chamber, as was the average relative humidity. It can therefore be surmised that not all of the mass that left the tube remained in the soil and that some was lost to the atmosphere in vapor phase. 




FIG. 8. Comparison between irrigation flux into sand, top soil and saline sand. Note that the flux calculation for the first $\mathbf{2 4}$ hours is affected by the initial filling of the tube and is subsequent calculations may be affected by tube swelling. Duplicates of each experiment are shown.

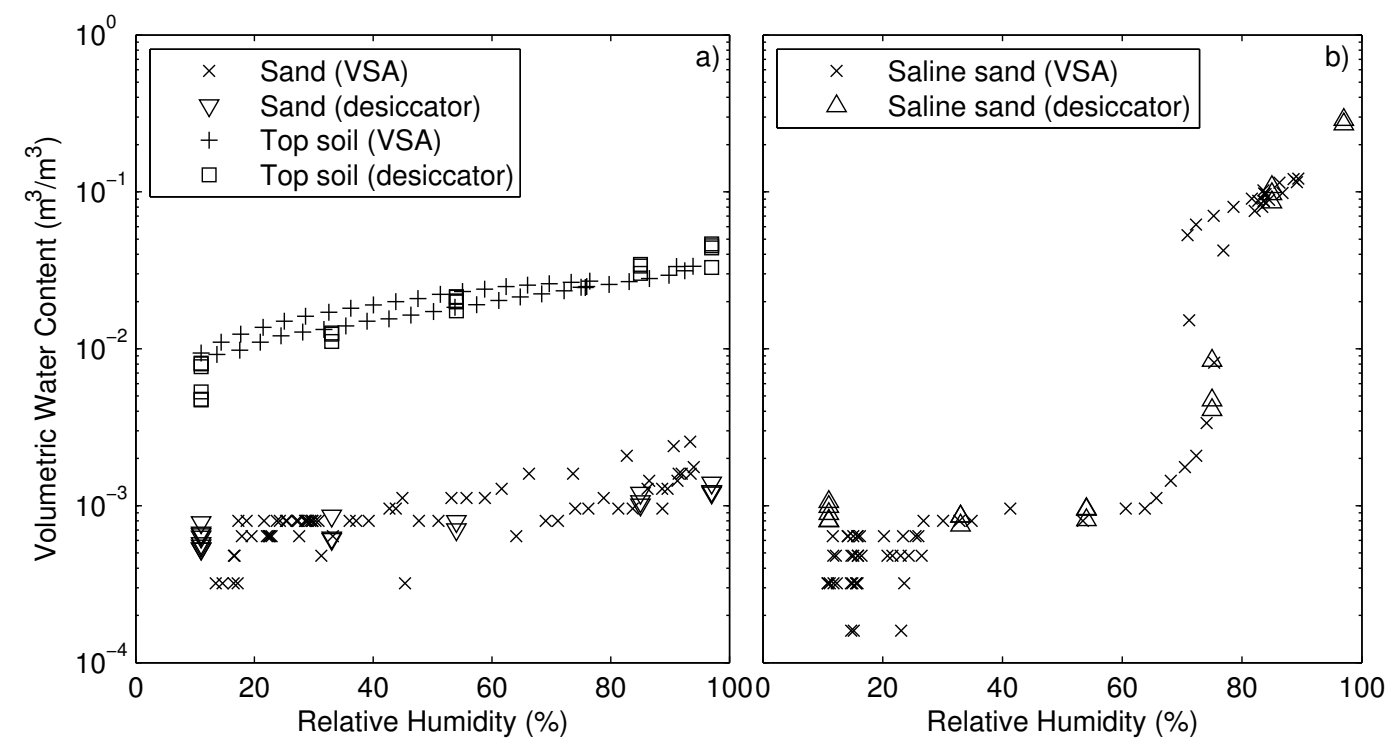

FIG. 9. Moisture sorption isotherm of a) sand and top soil and b) saline sand at $21^{\circ} \mathrm{C}$.

The moisture adsorption into sand is low thus it is unsurprising that vapor flow is significant. Figure ?? shows the results of the final set of soil box experiments, which compared the flux rate into marine sand to that into top soil and into 
salinized sand. All experiments used a soil depth of $10 \mathrm{~cm}$ and were conducted without a desiccant in the chamber. The flux rate into the top soil varied with time and decreased over the course of the test but remained higher than the flux into the sand throughout. The relative humidity in the chamber above the top soil began to increase around 50 hours after irrigation started, indicating that mass transfer into the atmosphere began to occur at this time. The flux rate into the salinized sand was higher than into the other two soils. As in the marine sand, the humidity in the chamber above the salinized sand quickly increased when irrigation was started, but stabilized at a lower value of approximately $80 \%$.

Figure ?? shows the moisture sorption isotherms calculated from desiccator and VSA experiments, and demonstrates that the differences in the humidity profiles in Figure ?? occurred due to the moisture sorption characteristics of the different soils. At low relative humidities sand adsorbs very little mass (Figure ??), thus the humidity profile in the sand increased quickly (Figure ??). The top soil adsorbs more mass at low humidity and the rate at which the mass increases with humidity is also greater (Figure ??). Consequently in the soil box test the increase in humidity was much slower than in sand as more of the flux out of the irrigation tube was adsorbed in the soil (Figure ??). The sorption isotherm for the saline sand is particularly interesting as the sand suddenly adsorbs more mass at a relative humidity of approximately $75 \%$. Such an increase in moisture adsorption due to the addition of salt is not unexpected as this has also been observed in the isotherms of pure sodium chloride (Foster and Ewing, 2000) and salted food products (Comaposada et al., 2000). Consequently, in the experiment in salinized sand, the humidity profile quickly increased to approximately $80 \%$, but flux from the tube remains high as moisture was being adsorbed into the soil. After one of the tests in top soil and one in saline sand samples were taken from from the soil to determine the soil water content. The initial water content in 


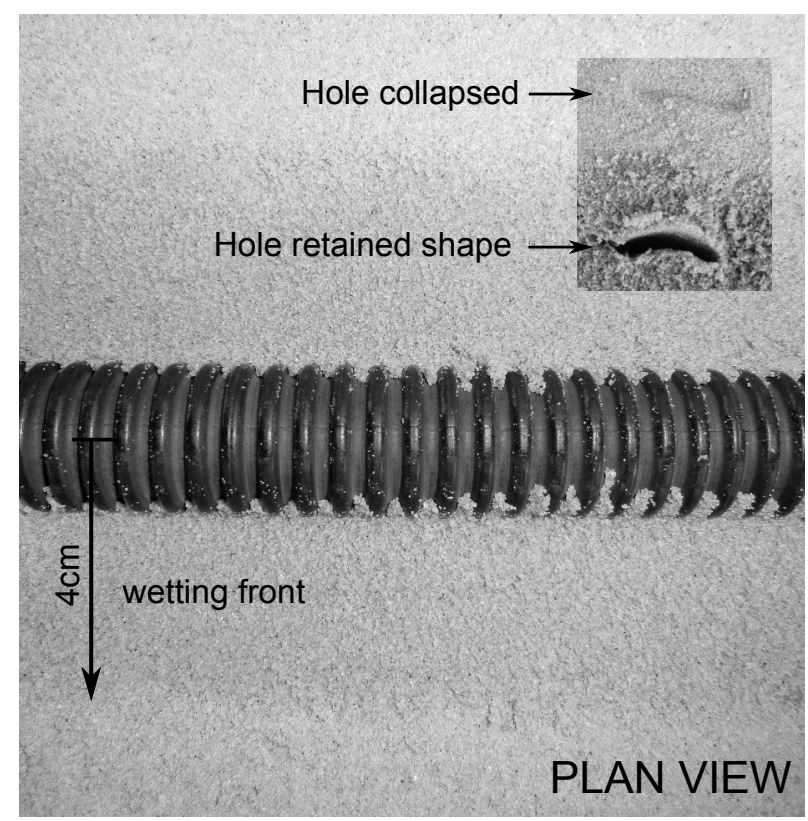

FIG. 10. Plan view of the wetting front in the saline sand, observable as a change in color and consistency. The hole left after inserting a rod collapsed ahead of the front but retained its shape in the wet sand.

both soils before the experiments started was close to $0 \mathrm{~m}^{3} / \mathrm{m}^{3}$ after oven drying. Close to the tube the water content was $0.037 \mathrm{~m}^{3} / \mathrm{m}^{3}$ in top soil and $0.12 \mathrm{~m}^{3} / \mathrm{m}^{3}$ in the saline sand. In the top $1 \mathrm{~cm}$ of the soil the water content was $0.026 \mathrm{~m}^{3} / \mathrm{m}^{3}$ in top soil and $0.001 \mathrm{~m}^{3} / \mathrm{m}^{3}$ in saline sand. Whilst the fluxes into both soils were of the same order of magnitude, the distribution of mass was different. The water content in the top soil was more distributed and remained below the residual water content of the soil (Figure ??) and within the range of water content measured during sorption experiments (Figure ??). However in the saline sand there was clear evidence of a wetting front (Figure ??) and the water content close to the membrane was greater than the residual value of $0.02 \mathrm{~m}^{3} / \mathrm{m}^{3}$ (Figure ??). After ten days the wetting front in saline sand extended approximately $4 \mathrm{~cm}$ either side of the tube, $3 \mathrm{~cm}$ above the tube and reached the bottom of the enclosing chamber. The absence of this front in the other two experiments is also significant, as it suggests that flow throughout these soils only occurred in the vapor phase. In 
the saline sand it is probable that some liquid flow occurred.

These results raise a number of further questions regarding how this system performs under field conditions. All of the experiments in soil have highlighted the importance of considering both the transport and condensation of the vapor phase. Thus, under field conditions, it is likely that factors that affect vapor behavior will affect the system performance. Such factors include diurnal temperature variations, which induce vapor flows (Phillip and de Vries, 1957), and soil heterogeneity, which will affect moisture sorption. It is also not clear how plant roots interact with water vapor. A small amount of work has shown that seeds absorb water vapor in the unsaturated zone (Wuest, 2007), but this work has not been extended to consider developed plants. The presence of plant roots is also likely to increase condensation in the soil. Further study is required to understand how plants interact with water supplied from a vapor source.

In some commercial pervaporation systems and in the experiments of QuiñonesBolaños et al. (2005a) air is swept over the surface of the PV membrane to maintain a high pervaporation flux. However, when the PV tube is buried soil (as in the experiments of Quiñones-Bolaños et al. (2005b) and in those presented in this paper) the humidity in the soil pores surrounding the membrane is high, resulting in a low flux across the membrane. Essentially, in these conditions, the flux is limited by the transport through the soil, away from the membrane rather than the membrane characteristics themselves. Thus if a plant roots are present and remove water from the soil the flux from the PV tube should increase. However, a significant increase in flux, would only be expected if the humidity in the soil was very low (e.g. below 50\%). Such low humidity corresponds to a soil water content retained by adsorption and well below the permanent wilting of a plant. Further work is required to establish whether such dry conditions continue to prevail when a PV membrane is used in a vegetated environment. 


\section{Summary and Conclusions}

To apply pervaporative irrigation systems successfully in the field it is important to predict the water that is likely to flow from the tube. The purpose of this study was to observe the flow rate in different controlled conditions so as to understand the fundamental processes that affect the interaction between the irrigation system and the soil. This work has shown that:

1. In humid air of approximately $100 \%$ relative humidity at $21^{\circ} \mathrm{C}$, the flux from the pervaporative tube is small; as the relative humidity decreases to $70 \%\left(21^{\circ} \mathrm{C}\right)$, the flux from the tube increases linearly. At lower humidities the flux may become limited by other factors and does not seem to increase further.

2. When buried in soil the tube is enclosed in an increasingly humid environment which depends on the soil sorption characteristics. Soil with high water sorption at low relative humidity increases the amount of condensation in the soil which results in an increased flux from the pipe. Hence, the addition of sodium chloride salt to sand $(16 \mathrm{~g} / \mathrm{kg})$ increased the flux rate by an order of magnitude. A moisture sorption isotherm is a useful predictor of this behavior.

3. Previous studies of pervaporative water transfer into soils assumed only liquid transport. This study has shown that vapor flow through dry soil is significant and affects the distribution of liquid water throughout the soil and the flux of water from the system. This vapor flow also leads to a loss of water to the atmosphere, thus failure to account for this process can lead to an over estimation of the soil moisture content. Hence, the availability of water for plant uptake from the soil cannot be predicted without considering vapor flow. 
4. As the humidity in the soil increases the flow from the tube decreases, and this increase in humidity occurs at low liquid moisture contents. Thus it is surmised that flux from the tube only occurs in very dry soil conditions. As little liquid water is available for plant uptake this raises an interesting question as to how the plants interact with the vapor flow emanating from this subsurface source. This question should be the subject of further study.

\section{Acknowledgements}

The authors acknowledge the financial support of the Engineering and Physical Sciences Research Council UK (EPSRC) provided via a PhD studentship and the in-kind support of DTI-r Ltd and Du Pont, without whom this work would not have been possible. We also gratefully acknowledge the funding for this work provided by the Research Partnership to Secure Energy for America (RPSEA) under Project Number 09123-11. Finally we would like to thank the reviewers for their helpful comments on this research.

\section{REFERENCES}

Agam, N. and Berliner, P.R. (2006). "Dew formation and water vapor adsorption in semi-arid environments - a review" Journal of Arid Environments, $65,572-590$

Beltrán, J. (1999). "Irrigation with saline water: benefits and environmental impact." Agricultural Water Management, 40(2-3), 183-194.

Bittelli, M., Ventura, F., Campbell, G., Snyder, R., Gallegati, F., and Pisa, P. (2008). "Coupling of heat, water vapor, and liquid water fluxes to compute evaporation in bare soils." Journal of Hydrology, 362, 191-205. 
Churaev, N. (2000). Liquid and vapor flows in porous bodies: surface phenomena. Gordon and Breach Science Publishers, Amsterdam, The Netherlands.

Comaposada, J., Gou, P., and Arnau, J. (2000). "The effect of sodium chloride content and temperature on the pork meat isotherms." Meat Science, 55, 291-295.

Dexter, A. and Richard, G. (2009). "Water potentials produced by oven-drying of soil samples." Soil Science Society of America Journal, 73(5), 1646-1651.

Feng, X. and Huang, R. (1997). "Liquid separation by membrane pervaporation: a review." Industrial and Engineering Chemical Research, 36, 1048-1066.

Foster, M. and Ewing, G. (2000). "Adsorption of water on the $\mathrm{NaCl}(001)$ surface. II. An infrared study at ambient temperature." Journal of Chemical Physics, 112(15), 6817-6826.

Haines, W. (1930). "Studies in the physical properties of soil. V. The hysteresis effect in capillary properties, and the modes of moisture distribution associated therewith." The Journal of Agricultural Science, 20(01), 97-116.

Hillel, D. (1998). Environmental soil physics 2nd Edition. Academic Press, California,USA

Jones, H. (2004). "Irrigation scheduling: advantages and pitfalls of plant-based methods." Journal of Experimental Botany, 55(407), 2427-2436.

Kelly, S. and Selker, J. (2001). "Osmotically driven water vapor transport in unsaturated soils." Soil Science Society of America Journal, 65(6), 16341641. 
Lebeau, M. and Konrad, J. (2010). "A new capillary and thin film flow model for predicting the hydraulic conductivity of unsaturated porous media." Water Resources Research, 46(12), W12554.

Lewis, J. and Sjöstrom, J. (2010). "Optimizing the experimental design of soil columns in saturated and unsaturated transport experiments." Journal of Contaminant Hydrology, 115, 1-13.

Pabby, A. K., Rizvi, S. S. H., and Sastre, A. M., eds. (2008). Handbook of Membrane Separations: chemical, pharmaceutical, food, and biotechnological applications. CRC Press, Boca Raton, USA.

Paul, D. (2004). "Reformulation of the solution-diffusion theory of reverse osmosis." Journal of Membrane Science, 241, 371-386.

Penov, I., Manolov, I., Alexiev, A., and Kavardziev, Y. (2011). "Salinisation in Bulgaria: institutional settings for soil conservation (a case study of Belozem village)." Land degradation and development, 22, 134-143.

Pereira, L. S., Oweis, T., and Zairi, A. (2002). "Irrigation management under water scarcity." Agricultural Water Management, 57(3), 175-206.

Phillip, J. and de Vries, D. (1957). "Moisture movement in porous materials under temperature gradients." Transactions, American Geophysical Union, $38(2), 222-232$.

Quiñones-Bolaños, E., Zhou, H., and Parkin, G. (2005a). "Membrane pervaporation for wastewater reuse in microirrigation." Journal of Environmental Engineering, 131, 1633-1643.

Quiñones-Bolaños, E., Zhou, H., Soundararajan, R., and Otten, L. (2005b). "Water and solute transport in pervaporation hydrophilic membranes to 
reclaim contaminated water for micro-irrigation." Journal of Membrane Science, 252(1-2), 19-28.

Quiñones-Bolaños, E. and Zhou, H. (2006). "Modeling water movement and flux from membrane pervaporation systems for wastewater microirrigation." Journal of Environmental Engineering, 132, 1011-1018.

Ruiz, T. and Benet, J. (2001). "Phase change in a heterogeneous medium: comparison between the vaporisation of water and heptane in an unsaturated soil at two temperatures." Transport in Porous Media, 44, 337-353.

Sadler, E., Evans, R., Stone, K., and Camp, C. (2005). "Opportunities for conservation with precision irrigation." Journal of soil and water conservation, $60(6), 371-379$.

Sumesh, P. and Bhattacharya, P. (2006). "Analysis of phase change during pervaporation with single component permeation." Colloids and Surfaces, $290,263-272$.

Wijmans, J. and Baker, R. (1995). "The solution-diffusion model: a review." Journal of Mebrane Science, 107, 1-21.

Wuest, S. (2007). "Vapour is the principal source of water imbibed by seeds in unsaturated soils." Seed Science Research, 17, 3-9.

TABLE 1. Properties of different soil types. Salinity was determined using a 1:5 soil:water volume ratio

\begin{tabular}{lccc}
\hline & Marine Sand & Saline Sand & Top Soil \\
\hline Packing Density $\left(\mathrm{kg} / \mathrm{m}^{3}\right)$ & 1600 & 1600 & 1000 \\
Salinity $(\mu \mathrm{S} / \mathrm{cm})$ & $1.4 \times 10^{1}$ & $9.0 \times 10^{3}$ & $2.7 \times 10^{2}$ \\
Organic Matter $(\%)$ & 0.05 & - & 8.91 \\
Carbon $(\%)$ & 0.03 & - & 5.08 \\
\hline
\end{tabular}

